\title{
Mixed-Initiative Approaches to Global Editing in Slideware
}

\author{
Darren Edge ${ }^{1}$, Sumit Gulwani ${ }^{2}$, Natasa Milic-Frayling ${ }^{3}$, Mohammad Raza $^{3}$, \\ Reza Adhitya Saputra ${ }^{1,4}$, Chao Wang ${ }^{1}$ \& Koji Yatani ${ }^{1,5}$ \\ ${ }^{1}$ Microsoft \\ Research \\ Beijing, China \\ ${ }^{3}$ Microsoft \\ Research \\ Cambridge, UK \\ ${ }^{4}$ University of \\ Waterloo \\ ${ }^{5}$ University of \\ Tokyo \\ \{daedge, sumitg, natasamf, a-moraza, chaowa\}@microsoft.com, radhitya@uwaterloo.ca, koji@iis-lab.org
}

\begin{abstract}
Good alignment and repetition of objects across presentation slides can facilitate visual processing and contribute to audience understanding. However, creating and maintaining such consistency during slide design is difficult. To solve this problem, we present two complementary tools: (1) StyleSnap, which increases the alignment and repetition of objects by adaptively clustering object edge positions and allowing parallel editing of all objects snapped to the same spatial extent; and (2) FlashFormat, which infers the leastgeneral generalization of editing examples and applies it throughout the selected range. In user studies of repetitive styling task performance, StyleSnap and FlashFormat were 4-5 times and 2-3 times faster respectively than conventional editing. Both use a mixed-initiative approach to improve the consistency of slide decks and generalize to any situations involving direct editing across disjoint visual spaces.
\end{abstract}

\section{Author Keywords}

Presentations; visual consistency; layout editing; snapping; programming by example; least-general generalization.

\section{ACM Classification Keywords}

H.5.2. Information interfaces and presentation: User Interfaces.

\section{INTRODUCTION}

Designing, delivering, and watching slide presentations are common aspects of professional life. As a modular authoring medium, slides constrain the problem of visual communication and afford flexibility in restructuring and reuse. However, this same modularity can result in weak connections between slides that are also visually inconsistent.

The theory of processing fluency proposes that aesthetic pleasure is based on perceptual processing, with high fluency associated with positive evaluations such as agreement [33]. For slide design, audience negativity toward visual disorder (e.g., clutter, inconsistency, misalignment) has been argues to result directly from reduced processing fluency [1].

\footnotetext{
Permission to make digital or hard copies of all or part of this work for personal or classroom use is granted without fee provided that copies are not made or distributed for profit or commercial advantage and that copies bear this notice and the full citation on the first page. Copyrights for components of this work owned by others than ACM must be honored. Abstracting with credit is permitted. To copy otherwise, or republish, to post on servers or to redistribute to lists, requires prior specific permission and/or a fee. Request permissions from Permissions@acm.org.
}

CHI 2015, April 18 - 23 2015, Seoul, Republic of Korea. Copyright is held by the owner/author(s). Publication rights licensed to ACM.

ACM 978-1-4503-3145-6/15/04 ..\$15.00

http://dx.doi.org/10.1145/2702123.2702551
Nancy Duarte, author of "Slide:ology" [10], recommends the reuse of slide layouts to help the audience anticipate where content will appear next (i.e., to increase processing fluency through repetition). Garr Reynolds, author of "Presentation Zen" [34], also recommends repetition, but of "certain design elements" rather than whole slide layouts. The use of slide templates, copied slide elements, and temporary grids can all help to avoid inconsistency problems, whether within slides (nonaligned elements appearing randomly placed), between slides (misaligned elements "jumping" on transition), or across the deck (related elements lacking consistent styling).

However, creating and maintaining visual consistency across slides is difficult when the desired layouts and styles are not known in advance. During the design of slide visuals, making systematic changes one element at a time is both repetitive and tedious, as well as making it difficult to refactor slide content into slide templates. Lack of support for the consistent redesign of elements repeated across slides is thus a major contributing factor to low processing fluency.

To address both the consumption problem of processing fluency and the authoring problem of repetitive styling, we present two complementary tools: (1) StyleSnap, for the automatic alignment of object edges within and across slides; and (2) FlashFormat, for the systematic restyling of related objects. Both are mixed-initiative systems [19] in which users collaborate with intelligent services to achieve their goals.

The intelligence of StyleSnap is through progressive hierarchical clustering of each of the four object edge positions (offsets from the left and top slide edges), in ways that increase alignment both within and across slides without introducing object overlaps or image distortions. Following an automated snapping process, the user can independently unsnap, merge, and style the resulting groups of objects that have been snapped to the same slide extent (i.e., all four corresponding edges share the same position values).

Conversely, the intelligence of FlashFormat comes after the user has directly supplied some examples of the repetitive edit they wish to apply more generally. It infers the leastgeneral generalization [32] of the example edits through the attribute values shared among all edited objects and the transformation performed on the edited objects. The next application of FlashFormat always extends the current edit to the smallest set of objects that can be generalized to by allowing the unshared attributes to vary freely. 
Since StyleSnap increases the number of shared attribute values throughout a slide deck (via both snapped object positions and propagated style changes), prior use of StyleSnap means that fewer example edits are required to cover all of the variance in the set of objects to be edited with FlashFormat. The two tools are thus complementary, although each has substantial value in standalone use.

In this paper, we first present a literature review on snapping and formatting, as well as the tools available in commonlyused graphical systems. We then present an examination of a PowerPoint slide-deck corpus, showing how the use of such templates in practice restricts their ability to create and maintain consistency. In the following sections, we describe the design and implementation of two tools - StyleSnap and FlashFormat - that address the limitations of conventional templates. We also present a user study of each, demonstrating their superiority over existing approaches in task performance time and user preferences. We then report the lessons learned from both systems and discuss improvements for future mixed-initiative editing systems. These are relevant to the category of slideware augmented in this paper, as well as any visual software that requires consistent styling across multiple pages (e.g., word processor documents), grids (e.g., spreadsheets), frames (e.g., when editing video overlays), or repeated visual spaces of any kind.

Overall, this work offers the following contributions:

1. Two approaches to selecting objects for systematic editing, based on: (a) similarity of objects' spatial extents as determined by clustering of their edge positions (StyleSnap); and (b) similarity of objects' attribute values as determined by the least-general generalization of example edits performed on other objects (FlashFormat).

2. Two approaches to simultaneously editing objects to reach a state of consistency, based on (a): snapping misaligned object edges into a reduced number of aligned positions, without introducing artifacts (StyleSnap); and (b) applying the least-general generalization of example edits to all objects selected for editing (FlashFormat).

\section{RELATED WORK}

Microsoft PowerPoint remains the most popular application in the category of "slideware" it established. PowerPoint also has a wealth of existing presentations available online, rich tools for the analysis of its XML-based documents, and an add-in framework for augmentation of the PowerPoint application. We therefore take PowerPoint as representative of slideware in the development and evaluation of our tools.

\section{Alignment of Document Objects}

Snapping is a commonly-used technique to guide objects into a state of alignment [5]. For example, PowerPoint 2013 provides visual line-guides and snapping behavior to support object alignment within a slide, as well as the traditional snapping of object edges to an underlying grid whose resolution can be customized. Object snapping can be extended in several ways, such as adaptively changing snapping behavior based on user input [26] or adaptively inserting motor space to support snap target differentiation [4]. Other approaches have addressed mouse-free snapping on surfaces and tabletops by using control-display gain [13], multi-touch [15], pen input [14], and the non-dominant hand [40]. None focus on cross-slide object alignment in slideware or alignment across disjoint visual spaces of any kind.

\section{Layout of Document Objects}

One common approach to achieving consistent, welldesigned layouts is to allow the user to select from a range of predesigned "templates" and enter content accordingly. The idea is that rather than making layout changes on individual slides (which can create inconsistencies), the user makes any changes on the template itself, automatically updating slides which adopt that template. In both PowerPoint 2013 and Keynote 6.2, such templates are provided in a special view called the Slide Master. This view defines slide layouts and styles through placeholders, which are also exposed to the user when they use menus to select a layout for a new slide or to update a slide to an alternative Slide Master layout. However, there are several usability problems with this approach. First, templates need to be decided in advance of slide creation rather than discovered through exploration on slides. Second, directly editing a slide object linked to a placeholder removes the ability to restyle that object through the Slide Master, unless the slide template is manually reapplied. When inspecting either an object or a slide, the inability to determine whether the placeholder links are intact makes it difficult to anticipate the scope of changes that are possible through slide templates. These problems belong to a more general category of risks that are known to reduce users' willingness to invest attention in abstraction use [6].

In the research domain, document analysis has been used to suggest layouts that satisfy criteria given by the user [24], such as logical structures of information to be visualized in presentation slides [38], or supports version management of multiple slide decks [9]. An alternative approach is to directly specify the structure of the desired document and allow the system to provide "styling as a service" as in the HyperSlides system for presentation prototyping [11].

One approach to automated layout is to consider it as a visual constraint satisfaction problem. Constraints express highlevel relationships between objects (e.g., text referencing a picture) or geometric structures (e.g., the sizes of all textboxes are the same). These constraints can be described as rules $[7,16,39]$. A major challenge of such rule-based systems is to anticipate all required rules. Another approach is to consider it as a problem of energy function optimization. This has been explored in the context of adaptive grids [20], driven by considerations such as goodness of template fit and micro-typography aesthetics, but is not always predictable for dynamic content such as news feeds. The concept of "conditional shapes and groups" can help to dynamically generate more flexible constraint systems [36], as can combinations of constraint- and force-based approaches [2]. 
While such automated layout systems are good for content that would not otherwise be "designed" or is yet to be designed, our tools specialize in the restyling of existing presentation content with a high degree of user control.

\section{Formatting of Document Objects}

The Slide Master is a form of indirect editing in PowerPoint and similar slideware. Another is the Format Painter, which allows all non-spatial attributes of a source object to be copied and "painted" onto destination objects, supporting reuse of object formatting in the slide view.

Macros, batch processing, history brushes, and graphical search and replace [22] are yet further ways in which users can capture and reuse action sequences. EAGER [8] is an early work using programming-by-example principles [29] to support efficient text data entry, but does not cover visual style changes. Abstract object selection and restyling is also possible with interactive machine learning [12], by inferring the user's desired scope based on patterns of selection and deselection (e.g., for file [35] and friend [3] selection).

For document editing, the LAPIS text editor [27] supports intelligent group selection and simultaneous reformatting of strings. An extension supports intelligent find-and-replace operations in text documents, grouping different string selection candidates by literal and semantic similarities [28].

\section{CORPUS ANALYSIS OF TEMPLATE USAGE}

We wanted to understand the extent to which the apparent usability problems of Slide Master templates were evident in examples of presentations downloaded from the Web. We built a corpus of over 8000 presentations using Bing web searches specifying the "ext:pptx" filter. The resulting slide decks were drawn from many fields including business, government, science, technology, and education. We used the Open XML SDK 2.5 [30] to parse these presentations and extract statistics on slides and objects. Table 1 shows the results from the 7663 successfully processed files.

We found that $88 \%$ of slides (24.7 out of 28.2 ) on average contained placeholder objects created by the Slide Master. We also found that the proportion of objects per slide that could actually be restyled through the Slide Master (without first resetting the slide to restore broken links, which loses any custom layout and styling of placeholders) was only $21 \%$ (1.1 out of 5.3) on average. That is to say, while the vast majority of slides ( $88 \%$ ) have the potential to be updated via the Slide Master, user editing behavior means that the vast majority of objects $(79 \%)$ require manual editing.

We conducted a second analysis to understand the degree to which objects shared the same or similar spatial extent on slides, corresponding to sharing the same or similar edge offsets (distances from the left and top slide edges). For each presentation, we first created a mapping from extents to objects, progressively varying the matching tolerance from 0 to 100 points in 10 points increments $(28 \mathrm{pt}=1 \mathrm{~cm})$. In each iteration, we grouped objects across slides whose edges were all located within a matching region of an existing extent

\begin{tabular}{l||c}
\hline Slide Statistics & Mean (SD) \\
\hline \hline \# of slides & $28.2(20.7)$ \\
\# of slides with placeholders unmodified & $19.0(17.5)$ \\
\# of slides with placeholders modified & $5.7(9.0)$ \\
\# of slides without placeholders & $3.5(7.8)$ \\
\hline Shape Statistics & Mean (SD) \\
\hline \hline \# of objects per slide & $5.3(6.1)$ \\
\# of objects created by user & $3.9(6.2)$ \\
\# of objects from unmodified placeholders & $1.1(0.9)$ \\
\# of objects from modified placeholders & $0.3(0.4)$
\end{tabular}

Table 1. Statistics on the number of slides and objects in 7,663 PowerPoint files we collected from the Internet.

(e.g., for the matching tolerance of 0 , we only grouped objects that had the exact same extent).

This analysis revealed a strong skew resulting from many small, often single-element groups. With exact matching (a tolerance of zero points), the average number of position groups was 35 and objects in the largest position group occurred on $82 \%$ of slides. All groups in the upper quartile (top 9 groups of 35) had objects occurring on at least $5 \%$ of slides. Since these cannot all be placeholder objects, they are likely to come from copy-and- paste used to ensure size, position, and style consistency for individual objects (and also slides). When the tolerance was 60 points (about $2 \mathrm{~cm}-$ a "near match" on all four object edges), the number of overall position groups halved (from 35 to 18 ). Objects in the largest position group occurred on $93 \%$ of slides and all position groups in the upper quartile (top 5 groups of 18) had objects occurring on at least $11 \%$ of slides.

In conclusion, there is ample opportunity to increase the positional consistency of objects by mapping "near match" objects into the exact same extent. If such a system could be developed, it would also create an opportunity to increase the style consistency of position groups by propagating style changes to all members of the associated group. Such a system would have greater restyling power than the Slide Master while also offering implicit object templates abstracted directly from slides, rather than explicit slide templates designed indirectly in a separate view. Many of the problems of template-based layouts could thus be avoided.

\section{STYLESNAP}

Following on from the previous corpus analysis, we designed a mixed-initiative tool called StyleSnap that can be applied whenever a slide deck has evolved into a state of misalignment or inconsistency. Our first goal was to develop a method to align objects across slides without introducing new problems, such as object overlap and image distortion. Our second goal was to develop a user interface that would allow a user to invoke StyleSnap, view the resulting groups of objects snapped to the same extent, then undo, merge, or modify these groups accordingly. Our implementation of these concepts was through an add-in for PowerPoint 2013. We now describe the high-level design of the StyleSnap user interface and details of the underlying snapping algorithm. 


\section{StyleSnap Interface}

Figure 1 shows the result of pressing the "StyleSnap" button in the PowerPoint ribbon menu. A side pane appears showing the resulting position groups - groups of objects across slides that have been mapped to the same position and size as a result of clustering and snapping edge values for the four edge types. Each group is listed showing its color (matching the color of the highlight boxes added to the corresponding objects), the number of objects in the group, a "Style Painter" icon for manually adding objects to the group and merging groups with one another, and a checkbox indicating whether the group is currently in its "Snap" state. Only non-singleton object groups are snapped by default, but the user can toggle snapping for both individual groups and for all objects. Snapped objects are shown with a solid border in their new extent; unsnapped objects with a dashed border in their original extent. If all objects were already in the same extent and unaffected by snapping, the Snap checkbox is disabled.

Clicking on any slide object or position group highlights the object, its position group in the side pane, and all other objects in the group. For efficient visual browsing of this position group, the system also gathers all slides containing highlighted objects and places them in a temporary "Selected" section at the start of the slide list, with other slides organized in an "Unselected" section (existing sections are recreated after StyleSnap tool use). The color of the selected group's highlight boxes also fully saturates for clear differentiation from the colors of other object groups. This visual feedback allows the user to quickly confirm whether the automatic snapping of the position group is desirable. When using the StyleSnap tool, any location, size, font, or other format changes to an object are propagated across all other objects in its group and made visible in real-time by simultaneous updates to all slides in the "Selected" section of the slide list.

The Style Painter is similar to the Format Painter but extends to position and size object attributes. Activating the Style Painter for a particular group by clicking the corresponding icon means that the next object or position group selected will automatically be merged with the active group.

Clicking the "Apply" button saves the changes to the deck and reverts to standard editing. Clicking "Discard" undoes the changes and reverts the deck to its pre-StyleSnap state.

\section{Snapping through Hierarchical Clustering}

We wanted to be able to "snap" objects into (a) fewer extents with (b) better alignment across slides. Each of these goals suggests a different approach. To reduce object extents, we could apply hierarchical clustering $[18,37]$ directly to object extents (since unlike, e.g., K-means, it does not require a prior choice for the number of clusters). However, this will not result in good cross-slide alignment if the extents of objects within slides are misaligned. There is also a sparsity problem - objects may all be sufficiently different that no two objects should be mapped to the same extent, even though any individual object edge may be close to the corresponding edges of many other objects.

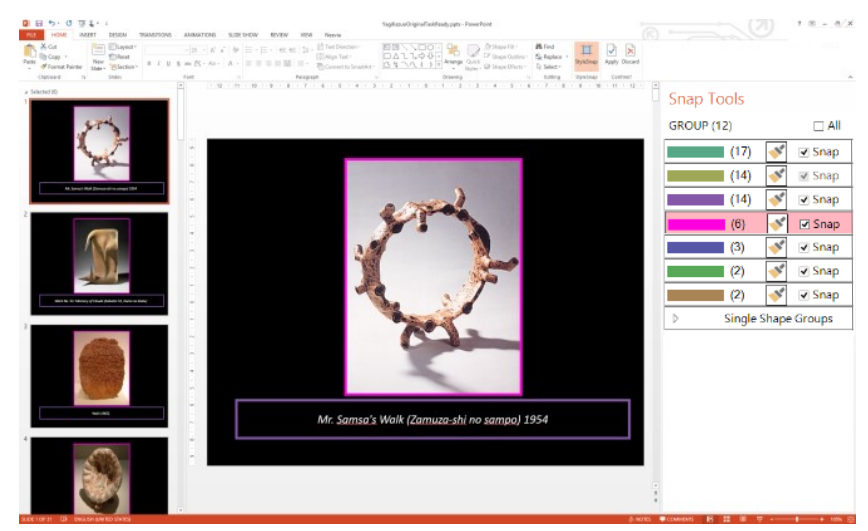

Figure 1. StyleSnap interface. The right pane shows all the groups whose objects are moved to the same position as a result of snapping. The user can manually undo and merge position groups, as well as simultaneously styling all objects in the same position groups with real-time feedback in the slide list.

This led us to a solution based on hierarchical clustering of not object extents, but individual object edges. That is, we independently perform hierarchical clustering of all the left, top, right, and bottom edge positions of the objects within a presentation, then apply the results back to those objects. The result of such hierarchical clustering for each of the four edge types is a hierarchy of depth $N$ where $N$ is the number of distinct edge positions. At level 1 in the hierarchy, there are $N$ clusters of 1 edge position. At level $N$ in the hierarchy, there is 1 cluster of $N$ edges, all snapped to the modal edge position. From level 1 to level $N$, the algorithm always merges the closest pair of clusters and snaps all edges in the new cluster to the new modal edge position.

We can determine the optimal clustering level for each edge type (left, top, right, and bottom) by an energy function that aims to balance similarity within and between clusters (i.e., to make close edge values the same while keeping distant edges separate). However, the naïve updating of object edge positions based on the optimum clustering of each edge type could easily cause problems in visual appearance, such as:

1. Object inversion, since independent edge clustering does not respect relative positions of opposing object edges;

2. Object overlap, since initially separated objects can be moved into a state in which their content regions overlap;

3. Image distortion, since images are especially sensitive to aspect ratio changes.

Given that we can identify and correct these problems only after position updates, our divide-and-conquer approach of clustering different edges independently is most suited to the agglomerative, bottom-up method of hierarchical clustering supported by the SLINK algorithm [37]. Overall, our snapping approach is summarized in Algorithm 1.

\section{Systematic Performance Evaluation of Snapping}

Even with around 500 objects (the $97^{\text {th }}$ percentile in our PowerPoint corpus), snapping completes in several seconds. To evaluate the degree to which our automated snapping matches human judgments, we recruited two professional 
software engineers to test its accuracy and reliability across a wide range of typical decks. They tested 80 presentations from our PowerPoint corpus, with varying numbers of slides (15 - 36; the lower and upper quartiles) as well as varying numbers of objects per slide $(2.5-6.0$ with mean 4.7$)$.

Table 2 shows how the number of singleton position groups (a mean of 53.6) forms a long-tail, as expected from the earlier corpus analysis. Problems resulting from snapping are well controlled in both group types, limited to overly shrinking objects, creating inconsistencies among sets of slide objects that were previously consistent (e.g., in size, spacing, or alignment), and breaking spatial relationships between objects (e.g., by moving arrows within diagrams). Shrinkage can be dealt with by some simple additional rules, but more complex rules or manual object grouping would be needed to resolve problems arising from object relationships. Overall, only 4.5 position group modifications on average were required to reach a satisfactory state of alignment.

\section{EVALUATION OF STYLESNAP}

We conducted two user studies to evaluate the performance of StyleSnap against two alternative approaches to crossslide alignment and styling: Repeat Editing and Slide Master. For the study tasks we selected a slide deck from our internet corpus with an average of 2 objects on each of 31 slides one from a Slide Master template, one added manually - with a balance of title-and-bullets and image-and-caption slides.

\section{Task: Cross-Slide Alignment of Misaligned Objects}

There were 14 slides containing one picture and one textbox used as a caption, with no perfect alignment of any pair of objects. The task was to align all four object edges of groups of images with similar aspect ratios and make the caption format the same across all 14 slides. For consistency, we defined target object groups for the 14 images based on three aspect ratios: 9 portrait images, 4 landscape, and 1 panoramic. We also set the target text format to be 20pt Red Italic Arial.

\section{Expert Performance Prediction}

To predict the expert performance of each technique, we adopted an approach akin to Keystroke Level Modelling (KLM) by constructing a task model for each system and quantifying its time parameters through a user study. Since these models do not account for switching costs, they represent predicted lower bounds on task completion time.

Repeat Editing: [Start time $T_{\text {re }}$ ] Make a duplicate of Slide 13 and use it as a template to align content from Slide 14. Delete unwanted objects and slides. [End time $T_{r e}$ ]

Reference task time $=T_{\text {re }} \times 13$ slides

Slide Master: [Start time $T_{1}$ ] Open the Slide Master view and create a layout with content placeholders in the desired positions and aspect ratios. Close the Slide Master. [End time $T_{s m 1}$ ] [Start time $T_{s m 2}$ ] Go to Slide 13 and update it to the new custom layout. Delete unwanted objects [End time $T_{s m 2}$ ]

Reference task time $=T_{s m 1} \times 3$ slide layouts (one per aspect ratio) $+T_{s m 2} \times 14$ slides (apply new layout to each)

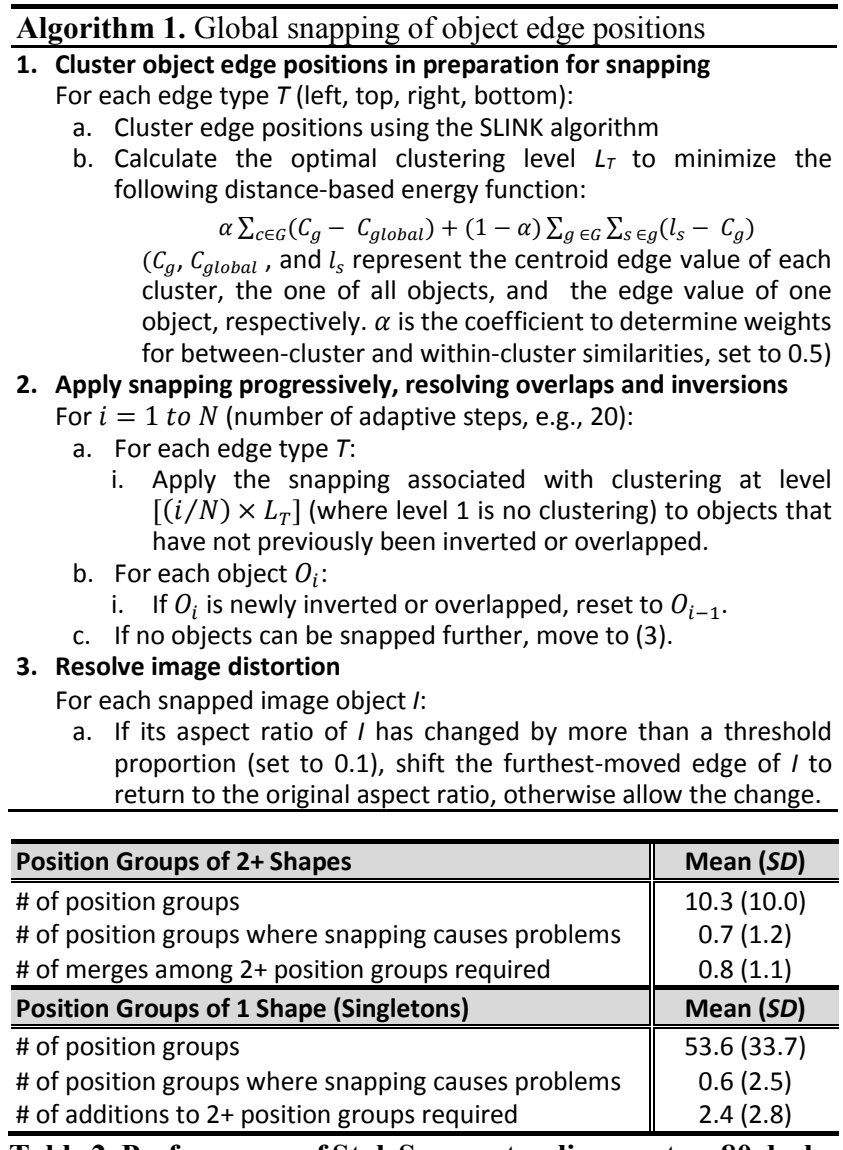

Table 2. Performance of StyleSnap auto-alignment on 80 decks.

StyleSnap: [Start time $T_{s s}$ ] Use the Style Painter to add the portrait image on Slide 28 to the position group of the portrait image on Slide 13. [End time $T_{s s 1}$ ] [Start time $T_{s s 2}$ ] Set the caption text to the target format. [End time $T_{s s 2}$ ]

StyleSnap groups the captions into a single group and images into groups with 4, 4, 2, 1, 1, 1, and 1 objects. Reaching three position groups for these images requires a maximum of eight changes using the Style Painter (one object at a time).

Reference task time $=T_{\text {ss } 1} \times 8$ group changes (to make 3 groups) $+T_{\text {ss } 2 \text { (applies to all captions at once) }}$

\section{Procedure}

We first explained and demonstrated each task before asking participants to repeat the procedure until they had mastered it. We used two such slides for our demonstration, with the task to make the layout and style of Slide 14 the same as Slide 13. We offered detailed instructions for each approach and allowed several timed trials until we did not observe large time variances. Participants' best times were recorded and used to calculate the reference task time (as is common when seeking to understand expert performance, e.g., in [40]).

We recruited 12 participants ( 6 male and 6 female, the average age of 25; PA1-PA12) from a local university. All were familiar with PowerPoint and fluent in English. We counterbalanced the condition order. Cash equivalent to $\$ 15$ USD in local currency was offered as compensation. 


\section{Expert Performance Results}

Table 3 summarizes the completion times of sub-tasks in each of the three techniques. Entering these times into our task models predict the average expert performance to be 353, 322, and 107 seconds with Repeated Editing, Slide Master and StyleSnap respectively. This prediction is highly promising and indicates that StyleSnap could substantially reduce the user effort of cross-slide alignment and styling. The results also show that updating any number of target slides with StyleSnap is comparable to refactoring a single slide with Slide Master, once the StyleSnap groups are correct and the Slide Master templates are created. Since 4.5 StyleSnap group merges are required to correct the snapping of a whole deck, on average, it also means that StyleSnap is about as fast at correcting alignment throughout this particular deck and refactoring 14 slides of a particular design (69s) as Slide Master is at creating a single template and refactoring just two slides (71s).

\section{Novice User Performance Measurement}

We also conducted a supplementary study with the same task set to measure the performance of novice users. The procedure was also the same except that participants were asked to modify all 14 slides in each task. This study therefore offers realistic performance observations on the three techniques that account for the effects of learning and fatigue. From the expert performance prediction results, we had the two hypotheses for this study: [HA-1] StyleSnap would be faster for global alignment than existing tools; and [HA-2] the perceived workload of global alignment tasks would be smaller with StyleSnap. StyleSnap training included 5-10 minutes of free exploration on a range of decks. Following the study tasks, participants completed a NASATLX questionnaire [17] for each approach. We recruited another eight participants for this study ( 5 male and 3 female, the average age of 25; PB1-PB8) from our research institute.

\section{Novice User Performance Results}

One-way repeated-measure ANOVA revealed a significant difference among techniques $\left(F_{(2,14)}=90.8, p<.0001, \eta_{\mathrm{p}}{ }^{2}\right.$ $=.93)$, with StyleSnap faster than the other two $(p<.0001$ for both), shown in Figure 2, supporting HA-1. Analysis of NASA-TLX responses found seven significant pairwise results as shown in Table 4, partially supporting HA-2.

While the cost of both Repeat Editing and the Slide Master scale linearly with the number of target objects, the cost of StyleSnap scales only with the number of corrections to groups containing target objects. The fewer groups that need to be corrected, the closer the performance of StyleSnap will get to a constant time cost, whatever the number of objects.

\begin{tabular}{l||c}
\hline StyleSnap Task for Expert Users: Completion Times & Mean (SD) \\
\hline \hline$T_{r e}:$ Refactor a target slide using Repeat Editing & $25.2(6.4)$ \\
$T_{s m 1}:$ Make a slide template using Slide Master & $43.2(9.6)$ \\
$T_{s m 2}:$ Refactor a target slide to a slide template using & $13.8(2.1)$ \\
Slide Master & \\
$T_{s s 1}:$ Find and add an object to a group using StyleSnap & $11.5(2.7)$ \\
$T_{s s 2}:$ Update all target slides using StyleSnap & $15.2(5.6)$ \\
\hline
\end{tabular}

Table 3. Sub-task completion time results in the expert performance user study.

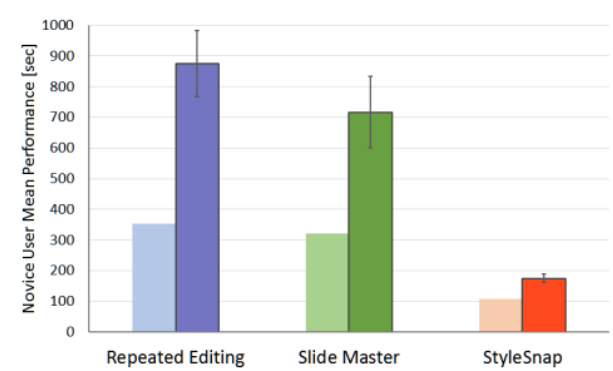

Figure 2. Predicted lower bounds on expert performance (bars without borders) and novice user mean performance time (bars with borders). Error bars represent $95 \%$ Confidence Intervals.

\section{Qualitative Discussion}

Participants in both studies unanimously preferred StyleSnap over Repeat Editing and Slide Master and many commented on how it would support their everyday authoring. The Slide Master was also widely criticized, e.g.: "If we edit using Slide Master, we really don't know what the final result will look like. After we edit it we should go back to see the result... if is not satisfying we should go back again to the slide master view... If we use StyleSnap we can see the result directly" (PA12).

The cost of automated snapping and manual modification was perceived to be relatively low. Participants described the "clustering" as "very efficient... it really helps in saving time" (PA4); "really useful" (PA8); " a great idea ... a simpler way to edit a lot of shapes" (PA9). Participants also appreciated being able to simultaneously edit all objects in a position group: "I can do quick edits for the whole content in the slides" (PA5); "You can do one 'style' and apply it everywhere fast" (PA2).

The study also highlighted areas for improvement. Several participants expressing initial confusion about the meaning of colors, suggesting use of semantic descriptors, icons, or thumbnails to make the mapping clearer. One participant also found the "shuffling" of the slide list distracting and another suggested using a drag-and-drop mechanism for Style Painting. From their free exploration with multiple decks, multiple participants also expressed a desire for the algorithm to automatically identify diagram-like collections of objects and group them for StyleSnap alignment purposes.

\begin{tabular}{|c|c|c|c|c|c|c|c|}
\hline \multicolumn{8}{|c|}{ StyleSnap Task for Novice Users: NASA-TLX Subjective Workload Results (in the format median [lower quartile, upper quartile]) } \\
\hline & Mental & Physical & Temporal & Performance & Effort & Frustration & Weighted overall \\
\hline Repeat Editing (RE) & $25.0[15.0,47.5]$ & $82.5[70.0,96.3]$ & $82.5[73.8,92.3]$ & $67.5[62.5,75.0]$ & $90.0[73.8,100.0]$ & $85.0[78.8,91.3]$ & $78.0[73.3,81.3]$ \\
\hline Slide Master (SM) & $47.5[40.0,57.5]$ & $47.5[33.8,58.8]$ & $45.0[27.5,57.5]$ & $22.5[8.8,36.3]$ & $52.5[25.0,55.0]$ & $52.5[36.3,66.3]$ & $43.8[35.8,55.0]$ \\
\hline StyleSnap (SS) & $30.0[18.8,66.3]$ & $22.5[18.8,31.3]$ & $17.5[13.8,21.3]$ & $15.0[5.0,16.3]$ & $27.5[25.0,33.8]$ & $20.0[8.8,30.0]$ & $25.0[22.7,29.3]$ \\
\hline Friedman test: $\chi^{2}(2)$ & $0.077(p=.96)$ & $11.6(p<.01)$ & $13.6(p<.01)$ & $10.2(p<.01)$ & $13.1(p<.01)$ & $14.0(p<.001)$ & $15.5(p<.001)$ \\
\hline Pairs with $p<.05$ & (none) & (none) & RE-SS & (none) & RE-SS, RE-SM & RE-SS, RE-SM & RE-SS, RE-SM \\
\hline
\end{tabular}

Table 4. NASA-TLX subjective workload results for novice users completing the StyleSnap task. Lower values are better. 
The study also surfaced tensions in the design of StyleSnap, which is predicated on the value of object alignment and style consistency throughout a slide deck. As one participant explained, "With StyleSnap I can easily design the layouts, especially if I want to change the same layouts, not manually edit the slides. But I think it will be difficult to change if on each slide the designs are different" (PA8). A different approach is required to make repetitive changes to objects that do not share the same position - this is the purpose of the complementary FlashFormat tool that we present next.

\section{FLASHFORMAT}

StyleSnap supports aligning objects across slides in ways that increase processing fluency, but it is not appropriate for repeated-object restyling when target objects are placed at different locations. FlashFormat offers the ability to apply global style changes with more flexible object selection.

FlashFormat is a programming-by-example system [29] that allows the user to perform repetitive formatting tasks in PowerPoint. The interface of FlashFormat is shown in Figure 3. It consists of only two buttons: "Start New Examples" and "FlashFormat". The user starts by clicking "Start New Examples" and then gives some examples of the formatting changes they would like to perform. They can then click "FlashFormat", at which point the system infers the leastgeneral generalization (LGG) from the given examples and applies to the rest of the document ("FlashFormat-all").

In Figure $3 \mathrm{~b}$ the user gives two examples of changing diamond shapes to the color yellow, for which the system infers the LGG that changes all diamond shapes to yellow (Figure 3c). The inferred generalization depends on the given examples; for instance, if the user instead desires to color any object with underlined text, then they may give examples with different shapes and colors (e.g., white diamond, white rectangle, gray rectangle) containing underlined text.

The inference performed by FlashFormat is based on the XML specifications of objects (using the OOXML file format), and hence covers all properties expressed in this specification. The system is based on a domain specific language for expressing transformations on XML structures, and a synthesis algorithm for inferring LGG programs within this language. In this work, we focus on the interaction model and usability studies on the tool. The underlying inference algorithm builds on prior work in program synthesis [32].

\section{An interactive and incremental generalization process}

The user can guide the system to the desired generalization in an interactive and incremental fashion. Since the system is conservative in the inference of the generalization, the user can give additional examples that are not very similar to cover their intended selection criteria. For example, if the user wants to color all objects containing underlined text and only gives examples of diamond shapes, then the system may infer the less general hypothesis of coloring only diamond shapes containing underlined text. However, the system is designed to incrementally accept examples, so at this point the user can give more examples through manual editing, and

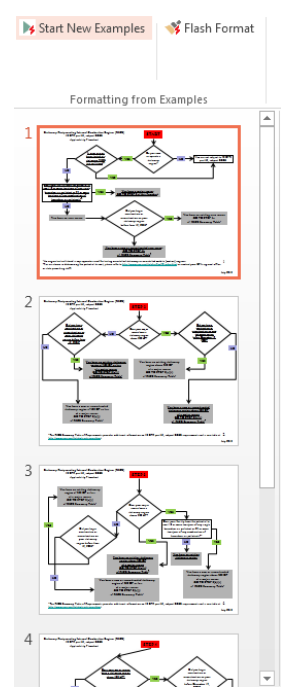

(a)

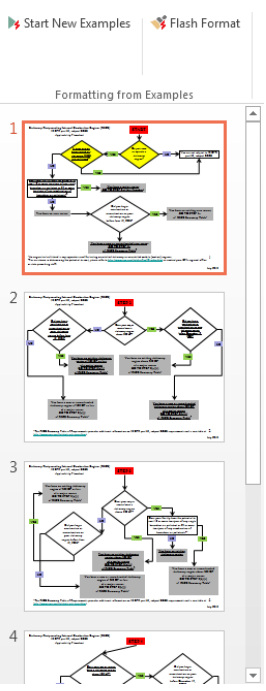

(b)

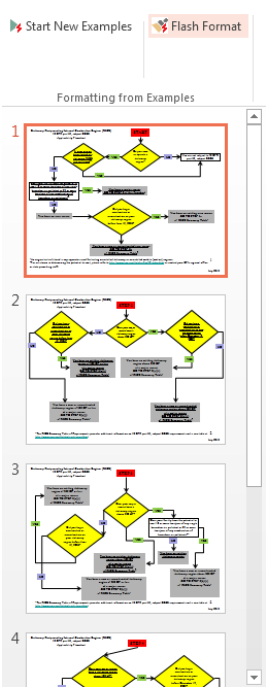

(c)
Figure 3. FlashFormat interface illustrating the coloring of all diamonds to yellow: (a) the user clicks "Start New Examples"; (b) she gives two examples of diamond shapes changing the color; (c) she clicks "Flash Format" to apply a least general generalization from the given example changes.

then FlashFormat again. The system will incorporate the new examples to expand the generality of the transformation.

The user can also backtrack if the system goes wrong at some stage. If the generalization inferred is not applicable to any other objects in the document, then the system applies the transformation to the closest matching objects, according to a similarity measure on the XML specification of objects. If this inference is inaccurate, then the user can undo the changes using the standard "undo" feature, and provide more examples to guide the system in the right direction.

The system also offers more restricted applications of the inferred transformations, which allows the user to verify the transformation before applying globally. After giving examples, the user can select a set of objects or slides and then click "FlashFormat" to only apply the transformation to the selected objects or slides ("FlashFormat-selected").

\section{EVALUATION OF FLASH FORMAT}

We conducted another user study to examine use of FlashFormat. More specifically, we had three hypotheses: [HB-1] Users would be able to achieve their desired global restyling through FlashFormat; [HB-2] experience of using FlashFormat would develop a sense of which examples to give, and [HB-3] using FlashFormat would be faster than standard editing tools across a range of tasks.

\section{Tasks: Cross-slide Shape Restyling}

We prepared a slide deck downloaded from the Internet. It contained a flowcharts spanning five slides using different shapes, such as rectangles and diamonds. Such diagrams are commonly encountered in slide presentations and cannot be restyled through the slide master because objects invariably occupy unique slide extents. Participants were asked to 
perform two changes specified by the experimenter either with FlashFormat or manually (use of Format Painter was allowed in this condition). In each task, we varied the number of objects to be restyled as well as the difficulty of example giving to create an approximately balanced workload per task. The participants were asked to make two systematic style changes in each trial, but were not allowed to change any other visual attribute or text content of the objects. After this controlled task, participants were given another slide deck and asked to perform global restyling as they liked. They were encouraged to use FlashFormat to make changes and were given five minutes for this part of the study.

The interface used in this study did not include visual feedback before clicking the FlashFormat button. Our intention was to study how well participants could understand the behavior of LGG without being influenced by the feedback design and test its effectiveness in a most basic form (any additional improvements on feedback would also generally favor the performance of FlashFormat).

\section{Procedure and Participants}

We first explained FlashFormat with two examples of slide decks and asked participants to perform global restyling tasks. The slide decks included easy and difficult cases for FlashFormat. This pre-task session was intended to make participants knowledgeable about the system and able to perform restyling without needing help from the experimenters. We provided explanations that choosing more and more diverse examples would lead to better results (referred to as "the golden rule"), but we did not force participants to do so. During each trial, we measured the performance time between when the participants started a task and when they confirmed all necessary changes on all specified objects. At the end of the study, participants were asked to describe their experience of FlashFormat and future improvements they would like, and to fill out a questionnaire.

We recruited 12 participants ( 8 male and 4 female, with average age 25; PB1-PB12) from our research institute. All were familiar with PowerPoint and fluent in English, and none of them participated in the first study. The same compensation was offered to all participants in this study.

\section{Results}

Figure 4 shows the mean performance time for each number of objects with the two techniques. Times in the manual editing condition varied in the range of 90-100 seconds, whereas FlashFormat times were constant at about 40 seconds. Two-way repeated measure ANOVA revealed that the technique had a significant main effect $\left(F_{(1,11)}=45.5, p\right.$ $\left.<.0001, \eta_{\mathrm{p}}{ }^{2}=.81\right)$. The interaction of the technique and the task was also significant $\left(F_{(2,22)}=4.40, p<.05, \eta_{\mathrm{p}}{ }^{2}=.29\right)$. The post-hoc analysis on the interaction effect confirmed that FlashFormat was significantly faster than the manual editing condition $(p<.0001)$. This quantitative result demonstrates substantial improvements on global restyling over existing editing tools and methods, supporting our hypothesis HB-3.

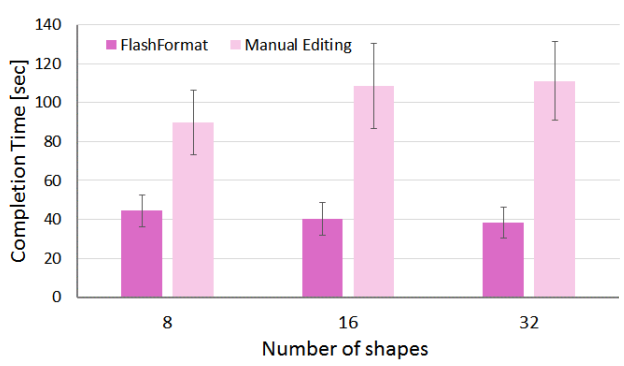

Figure 4. Mean performance time in the second study. The error bars represent $95 \%$ Confidence Intervals.

\begin{tabular}{l|l}
\hline Questions & Mean response (SD) \\
\hline \hline $\begin{array}{l}\text { I could always give appropriate examples to reach } \\
\text { my desired end state. }\end{array}$ & $5.1(1.6)$ \\
\hline $\begin{array}{l}\text { I could anticipate the effects of FlashFormat before } \\
\text { actually doing it. }\end{array}$ & $4.6(1.4)$ \\
\hline $\begin{array}{l}\text { It is necessary to anticipate the effects of } \\
\text { FlashFormat to successfully use the tool. }\end{array}$ & $5.8(1.2)$ \\
\hline $\begin{array}{l}\text { It is annoying to repeatedly undo unwanted effects } \\
\text { from FlashFormat. }\end{array}$ & $4.3(2.1)$ \\
\hline $\begin{array}{l}\text { I would prefer to use FlashFormat rather than make } \\
\text { repetitive changes one-by-one. }\end{array}$ & $6.5(0.8)$ \\
\hline
\end{tabular}

Table 5. Responses of the post-experimental questionnaire. (1: strongly disagree - 7: strongly agree)

Table 5 shows the questionnaire results. Participants responded positively to their experience with FlashFormat, unanimously agreeing that FlashFormat was preferable to manual editing. As with StyleSnap, many participants commented on how it would support their regular authoring practices, especially with regard to diagram formatting. We further examined the qualitative study data to understand the reasons for these positive results.

\section{Qualitative Analysis}

In the pre-task session, participants exhibited a nearuniversal tendency to give one example, repeatedly FlashFormat-all until the point of over-generalization, undo, and repeat. That is to say, participants did not follow the golden rule despite being reminded after each undo action. However, as the system repeatedly failed to produce their intended generalization from a single example, participants began giving more examples. At first, these were typically two examples given on the first two slides where they were applicable, but we observed a gradual shift to a more systematic selection of diverse examples, as initially suggested in the golden rule. Not only did participants learn to follow the golden rule through experience, they also learned for themselves what it means for examples to be diverse and how to give enough depending on the situation:

"Doing things automatically is good but sometimes I need to find out the differences of the shape, for example this is red and this is orange so I know I have to give two examples, one on the red and another on the orange, to let the system know that I want to change all the shapes no matter what the color is." (PB2)

"If you want to change the styles for all slides, you have to review all the slides first. For me it should be: first review all the slides, and then pick up all the different parts... different stuff in the same parts you want to change, and then change the different properties, and go ahead and FlashFormat and it should work." (PB8) 
Overall, this suggests that repeated feedback from applying the LGG of self-selected formatting examples is sufficient to promote self-discovery of the optimum example-giving and generalization strategy. Our questionnaire results also support this, showing that participants agreed that they could provide examples and anticipate the effect. Thus, we concluded that HB-1 and HB-2 are also supported.

Nevertheless, the learning process could still benefit from additional guidance. One participant highlighted how before starting new examples, they "always forget to press the button" (PB12). Another participant described how while giving examples, they "sometimes feel lost about what to do next should I choose more examples, or should I apply FlashFormat first?" (PB1). Finally, both before and after applying FlashFormat-all, several participants reported the need for better feedback about which objects either would be or had been changed. Suggestions for improvements included highlighting the scope of objects that would be changed next, highlighting the differences of objects that share some attributes with the selected object to guide example selection, learning from multi-object examples where the relative changes are important from a graphic design perspective, and removing the need to press a start button, either by showing a history of recently edited attributes that will be applied or suggesting multiple transformations from a single example.

Even without these suggested changes, the approach fares favorably against alternatives. Compared with the PowerPoint Format Painter, it was found to "work more efficiently" (PB3) and to be "much faster" (PB12) and "much more powerful" (PB4) because it can "work globally" (PB4) and "between slides" (PB5) without overwriting all attributes (PB4). FlashFormat also "has some features Slide Master does not provide" (PB7), such as the ability to work on groups of objects that do not share the same location. In this respect, FlashFormat also surpasses the restyling power of StyleSnap, in which object groups are formed only by shared extents.

\section{OVERALL DISCUSSION}

We presented two complementary tools for quickly making global changes that can improve the visual consistency of slide decks. We now synthesize the limitations, lessons, and future work from the design and evaluation of these two tools.

One limitation of the current work is our focus on the design of slide visuals only, and not of the underlying presentation material [23] or narrative structure [31]. While the balance of presentation preparation time should arguably be in favor of content and story, any time saved on styling slide visuals could conceivably be transferred to these other activities.

Another limitation is our sole use of PowerPoint for corpus analysis and prototyping. Since all slides and slideware are structurally similar, we expect our proposed solutions to generalize to other slideware and their associated slides. As with generalization to other domains (e.g., vector-based graphical editing), this has not yet been demonstrated.
We have also already noted that StyleSnap does not preserve size and spacing constraints within a slide. Constraint-based reasoning as used for single layout beautification [41] could provide a solution. Similarly, a limitation of FlashFormat is that imperceptible differences in attribute values are still viewed as differences by the system, leading to potential mismatches between user expectations and action outcomes.

Finally, we have evaluated our two tools independently rather than as a single system, such that we may evaluate their individual value. We hope to combine the functionality of the two tools into a single system in future work, taking into account the lessons discussed next.

\section{Lesson 1: Suggest generalizations from single edits}

One of the advantages of StyleSnap over FlashFormat is that users can confidently edit all of the objects in a position group at once rather than after giving several examples. One of the advantages of FlashFormat over StyleSnap is that object groups can be formed from any set of shared attributes, not just edge positions. Future work should investigate how to achieve both high predictability and flexibility from single examples. This could be achieved by suggesting multiple candidate transformations after each object edit, or suggesting snapping results for attribute values beyond edge positions (e.g., to create small, consistent sets of colors and font sizes) after each object selection. Users could thus make progress by confirming the desirability of candidate edits.

Lesson 2: Support state preservation as well as propagation In both StyleSnap and FlashFormat, there were times when the user already had an example of their desired end state but were forced to recreate it for the benefit of the tool. In StyleSnap, this was because the snapped object groups represented the modal values of the clustered edges rather than the extent of a specified object. In FlashFormat, this was because only the edited attributes of an object contribute to the inferred example, not any other existing attributes. Future work should explore how to give examples of both the "change to" and "keep as" variety on a per-attribute basis, without requiring an enumeration of all object attributes.

\section{Lesson 3: Show the scope of prospective changes}

In StyleSnap, the real-time feedback from the combination of object highlights and the Selected slide section gave users confidence in the scope of their changes before and as they were making them. However, this feedback also created much visual noise for dense slides and much scrolling of the slide list for large numbers of selected objects. As literature suggests the importance of feedback in this type of systems [29], future work should explore alternative feedback strategies for tools like both StyleSnap and FlashFormat.

\section{Lesson 4: Incorporate design patterns and principles}

StyleSnap makes the layout of objects consistent across slides, but it does not give any guidance about the desirability of those layouts. Similarly, FlashFormat can make largescale changes easily, but provides no feedback about the desirability of those changes (e.g., on the contrast between text and its background image following a global change to 
the color of overlaid captions). Future work should explore how to resolve aesthetic issues through assisted layout and styling that considers factors such as visual balance [25] and mood [21]. Supporting consistency not just within sets of user-created visuals, but with external design patterns and principles, remains a significant research challenge.

\section{REFERENCES}

1. Abela, A.V. (2008). Advanced presentations by design. Pfeiffer.

2. Ali, K., Hartmann, K., Fuchs, G. \& Schumann, H. (2008). Adaptive layout for interactive documents.

SmartGraphics'08, 247-254.

3. Amershi, S., Fogarty, J. \& Weld., D. (2012). Regroup: interactive machine learning for on-demand group creation in social networks. CHI'12, 21-30.

4. Baudisch, P., Cutrell, E., Hinckley, K. \& Eversole, A. (2005). Snap-and-go: helping users align objects without the modality of traditional snapping. CHI'05, 301-310.

5. Bier, E.A. \& Stone, M.C. (1986). Snap-dragging. SIGGRAPH'86, 233-240.

6. Blackwell, A.F. (2002). First steps in programming: A rationale for attention investment models. Human Centric Computing Languages and Environments, 2002.

7. Borning, A., Lin, R.K.H \& Marriott, K. (2000). Constraintbased document layout for the Web. Multimedia Syst. 8(3), 177-189.

8. Cypher, A. (1991). EAGER: programming repetitive tasks by example. CHI'91, 33-39.

9. Drucker, S.M., Petschnigg, G. \& Agrawala, M. (2006). Comparing and managing multiple versions of slide presentations. UIST'06, 47-56.

10. Duarte, N. (2008). Slide:ology: The art and science of creating great presentations. O'Reilly Media.

11. Edge, D. Savage, J. \& Yatani, K. (2013). HyperSlides: dynamic presentation prototyping. CHI'13, 671-680.

12. Fails, J.A. \& Olsen Jr., D.R. (2003). Interactive machine learning. IUI'03, 39-45.

13. Fernquist, J., Shoemaker, G. \& Booth, K. S. (2011). "Oh snap"-helping users align digital objects on touch interfaces. INTERACT'11, 338-355.

14. Frisch, M., Kleinau, S., Langner, R. \& Dachselt, R. (2011). Grids and guides: multi-touch layout and alignment tools. CHI'11, 1615-1618.

15. Frisch, M., Langner, R. \& Dachselt, R. (2011). Neat: a set of flexible tools and gestures for layout tasks on interactive displays. ITS'11, 1-10.

16. Graf, W.H. (1998). Constraint-based graphical layout of multimodal presentations. Readings in intelligent user interfaces, Morgan Kaufmann, 263-285.

17. Hart, S. G., \& Staveland, L. E. (1988). Development of NASA-TLX: results of empirical and theoretical research. Human mental workload, 1(3), 139-183.

18. Hastie, T., Tibshirani, R. \& Friedman, J.J.H. (2001). The elements of statistical learning. Springer.

19. Horvitz, E. (1999). Principles of mixed-initiative user interfaces. CHI'99, 159-166.

20. Jacobs, C., Li, W., Schrier, W., Bargeron, D. \& Salesin, D. (2003). Adaptive grid-based document layout.

SIGGRAPH'03, 838-847.
21. Jahanian, A., Liu, J., Lin, Q., Tretter, D., O'Brien-Strain, E., Lee, S.C., Lyons, N. \& Allebach, J. (2013).

Recommendation system for automatic design of magazine covers. IUI'13, 95-106.

22. Kurlander, D. \& Bier, E. (1988). Graphical search and replace. SIGGRAPH'88, 113-120.

23. Liu, Y., Edge, D. \& Yatani, K. (2013). SidePoint: a peripheral knowledge panel for presentation slide authoring. CHI'13, 681-684

24. Lok, S. \& Feiner, S.K. (2001). A survey of automated layout techniques for information presentations. SmartGraphics'01, 61-68.

25. Lok, S., Feiner, S.K. \& Ngai, G. (2004). Evaluation of visual balance for automated layout. IUI'04, 101-108.

26. Masui, T. (2001). HyperSnapping. HCC'01, 188-194.

27. Miller, R.C. \& Myers, B.A. (2002). Multiple selections in smart text editing. IUI' 02, 103-110.

28. Miller, R.C. \& Marshall, A.M. (2004). Cluster-based find and replace. CHI'04, 57-64.

29. Myers, B. A. (1992). Demonstrational interfaces: a step beyond direct manipulation. Computer 25(8), 61-73.

30. Open XML SDK 2.5. http://msdn.microsoft.com/enus/library/office/bb448854.aspx

31. Pschetz, L., Yatani, K. \& Edge, D. (2014). TurningPoint: narrative-driven presentation planning. CHI'14.

32. Raza, M., Gulwani, S. \& Milic-Frayling, N. (2014). Programming by example using least general generalizations. AAAI.

33. Reber, R., Schwarz, N., \& Winkielman, P. (2004). Processing fluency and aesthetic pleasure: is beauty in the perceiver's processing experience?. Personality and social psychology review, 8(4), 364-382.

34. Reynolds, G. (2012). Presentation Zen: simple ideas on presentation design and delivery. New Riders.

35. Ritter, A. and Basu, S. (2009). Learning to generalize for complex selection tasks. IUI'09, 167-176.

36. Schrier, E., Dontcheva, M., Jacobs, C., Wade, G. \& Salesin, D. (2008). Adaptive layout for dynamically aggregated documents. IUI'08, 99-108.

37. Sibson. R. (1973). SLINK: an optimally efficient algorithm for the single-link cluster method. The Computer Journal $16(1), 30-34$.

38. Watanabe, T. \& Hanaue, K. (2013). Composition support of presentation slides based on transformation of semantic relationships into layout structure. Multimedia Services in Intelligent Environments, 25, 155-181.

39. Weitzman, L. \& Wittenburg, K. (1996). Grammar-based articulation for multimedia document design. Multimedia Systems, 4(3), 99-111.

40. Wigdor, D., Benko, H., Pella, J., Lombardo, J. \& Williams, S. (2011). Rock \& rails: extending multi-touch interactions with shape gestures to enable precise spatial manipulations. CHI'11, 1581-1590

41. Xu, P., Fu, H., Igarashi, T. \& Tai, C-L. (2014). Global beautification of layouts with interactive ambiguity resolution. UIST'14, 243-252. 